\title{
Physical Interpretation of the Dirac Neutrino with Electromagnetic Mass*
}

\author{
Héctor Torres-Silva
}

Escuela de Ingeniería Eléctrica Electrónica, Universidad de Tarapacá, Arica, Chile.

Email: htorres@uta.cl

Received April $6^{\text {th }}, 2013$; revised May $6^{\text {th }}, 2013$; accepted May $14^{\text {th }}, 2013$

Copyright (C) 2013 Héctor Torres-Silva. This is an open access article distributed under the Creative Commons Attribution License, which permits unrestricted use, distribution, and reproduction in any medium, provided the original work is properly cited.

\begin{abstract}
The usual choice of an orthogonal set of four plane-wave solutions of the free-particle Dirac equation does not lend itself readily to direct and complete physical interpretation in the case of Dirac neutrino particle. A different choice of solutions can be made which yields a direct physical interpretation at all energies. Besides the separation of positive and negative energy states there is a further separation of states for which the spin is respectively parallel or antiparallel to the direction of the momentum vector. This can be obtained from the Maxwell's equation without charges and current in the $\boldsymbol{E} \| \boldsymbol{H}$ configuration. Scenery of our study is at high temperature $T$ where coexist electron-positron pair and neutrino-antineutrino pair, (i.e., $T \geq 1 \mathrm{MeV}$ ). Taking into consideration the possibility of negative mass, we can describe the observed behavior of antimatter in response to electromagnetic fields by assuming that the anti Dirac neutrino has a negative mass, so a new causal symmetry can be obtained.
\end{abstract}

Keywords: Chiral Neutrino; Dirac Equation; Maxwell System

\section{Introduction}

Neutrino electromagnetic properties, which are the main subject of this paper, are of particular importance because they provide a kind of bridge to "new physics" beyond the standard model. In spite of reasonable efforts in studies of neutrino electromagnetic properties, up to now there is no experimental confirmation in favour of non vanishing neutrino electromagnetic characteristics. [1]. The group of elementary particles called neutrinos (denoted by the greek letter " $v$ ") belongs to the lepton family (together with electrons, muons and tauons). They are electrically neutral and travel close to the speed of light. There are three (known) flavours, or types, of neutrinos, electron neutrino $\left(v_{e}\right)$, muon neutrino $\left(v_{\mu}\right)$ and tau neutrino $\left(v_{\tau}\right)$, all with their corresponding antiparticles. All neutrino interactions are mediated by the weak nuclear force (and of course gravitation) [2].

Electron neutrinos, muon neutrinos, and tau neutrinos that can morph into one another. For example, when cosmic rays strike the atmosphere, they create particles called muons that decay much as neutrons do, to produce muon neutrinos [3]. The muon neutrinos can then "oscil-

\footnotetext{
*This work has been supported by the Proyecto Mayor 8731-13, 2013
} of the Universidad de Tarapacá, Chile. late" or "mix" into other flavors before reaching Earth, as observed in 1998 by physicists using a detector SuperKamiokande in Japan. Electron neutrinos from the sun also change flavor, as physicists at the Sudbury Neutrino Observatory in Canada showed in 2001 [4].

This means that neutrinos interact weakly via the electromagnetic force at all, and this makes them very difficult to detect for us. The cross section of a typical neutrino is very small, and it is therefore able to pass though ordinary matter very easily [5].

The mass of the neutrinos is not well known. Measurements of the speed of neutrinos have failed to show that they move slower than the speed of light, which indicate no or an extremely small mass. However, evidence for neutrino oscillation demands that neutrinos have masses [6]. Neutrino oscillation enables neutrinos of one flavour to change into another flavour. This happens with a certain probability that is proportional to the difference of the square of the masses. Since these flavour oscillations have been observed, neutrinos are believed to have very small but non zero rest masses and that neutrinos generally are relativistic (their energy is much larger than their rest mass) [7].

In the course of the recent development of knowledge 
on neutrino mixing and oscillations, supported by the discovery of flavor conversions of neutrinos from different sources, non-trivial neutrino electromagnetic properties seem to be very plausible [8].

In particular, right-handed neutrinos constitute a common new physics proposal, usually linked to the generation of neutrino masses. This is particularly interesting nowadays, ever since we gathered compelling evidence that neutrinos do have masses, that they lie well below the other fermions' ones, and that their mixing patterns differ extraordinarily from those of the quark sector (for a review on the matter of neutrino masses see, for example, [1]). The most straightforward way to construct a mass term for the neutrinos within the SM is just to rely on the Higgs mechanism, and so to write the corresponding Yukawa couplings; for that aim, one needs some fermionic felds which carry no SM charge: righthanded neutrinos. However, we do not know whether neutrinos are Dirac or Majorana.

If they are Dirac, the smallness of the neutrino mass scale remains unexplained, for it would be just a product of the smallness of the corresponding Yukawa couplings. In order to provide such an explanation, many models and mechanisms have been proposed: in the so-called see-saw models, the lightness of the neutrino mass scale is a consequence of the heaviness of another scale. For instance, this scale is the lepton-number-violating (LNV) Majorana mass of the extra right-handed neutrinos in type I see-saw [9]. Although some of these proposals do not require right-handed neutrinos, for the sake of generality it is a good idea to consider their possible involvement in the generation of neutrino masses.

The existence of a relic sea of neutrinos is a generic feature of the standard hot big bang model, in number only slightly below that of relic photons that constitute the cosmic microwave background (CMB). This cosmic neutrino background (CNB) has not been detected yet, but it presence is indirectly established by the accurate agreement between the calculated and observed primordial abundances of light elements, as well as from the analysis of the power spectrum of CMB anisotropies and other cosmological observables. In this introduction we will summarize the evolution and main properties of the CNB.

Neutrinos are kept in equilibrium at high temperatures by reaction involving radiation and weak nuclear force. To keep the analysis as simple as possible, consider the reaction where a strong electromagnetic wave (photon) in its own reference frame, is split into two chiral photons producing a electron-positron pair which collide to form a tau neutrino-antitau neutrino pair

$$
\gamma \rightarrow v+\bar{v} \Leftrightarrow e^{-}+e^{+} .
$$

At sufficiently high temperature the reverse reaction is also possible. Hence the neutrinos are held in equilibrium.
While coupled to the rest of the primeval plasma (relativistic particles such as electrons, positrons, and photons), neutrinos had a momentum spectrum with an equilibrium Fermi-Dirac form with temperature $T$

$$
f_{e q}(p, T)=[\exp ((p) / T)+1]^{-1}
$$

As the universe cools, the weak interaction rate $\Gamma_{v}=\left\langle\sigma_{v} n_{v} c\right\rangle$ falls below the expansion rate and one says that neutrinos decouple from the rest of the plasma. $\sigma_{v} \propto G_{F}^{2}$ is the cross section of the electron-neutrino processes with $G_{F}$ the Fermi constant and $n_{v}$ is the neutrino number density. An estimate of the decoupling temperature $T_{d e c}$ can be found by equating the thermally averaged value of the weak interaction rate $\Gamma_{v}$ with the expansion rate given by the Hubble parameter $H$ (the Hubble time $H^{-1}(t)$ represents an upper limit to the age of the Universe).

$$
H=\sqrt{8 \pi / 3} \sqrt{\rho} / M_{P}
$$

Here $\rho \propto T^{4}$ is the total energy density, dominated by relativistic particles, and $M_{P}=1 / G^{1 / 2}$ is the Planck mass. If we approximate the numerical factors to unity, with $\Gamma_{v} \approx G_{F}^{2} T^{5}$ and $H \approx T^{2} / M_{P}$, we obtain the rough estimate $T_{\text {dec }} \approx 1 \mathrm{MeV}$ (see Figure 1).

Although neutrino decoupling is not described by a unique $T_{d e c}$, it can be approximated as an instantaneous process. The standard picture of instantaneous neutrino decoupling is very simple (see e.g., [2]) and reasonably accurate. In this approximation, the spectrum in $f_{e a}(p, T)$ is preserved after decoupling, because both

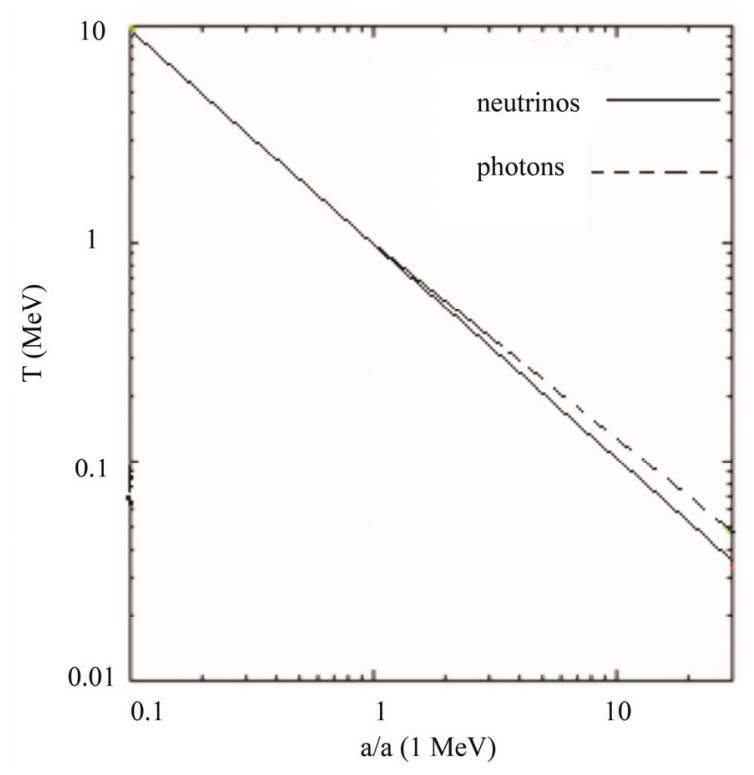

Figure 1. Photon and neutrino temperatures during the process of annihilations: evolution of their decrease with the expansion $a$ of the universe which is normalized to $a$ (1 MeV). 
neutrino momenta and temperature redshift identically with the expansion of the universe. Shortly after neutrino decoupling the temperature drops below the electron mass, favouring $e^{ \pm}$annihilations that heat the photons. If one assumes that this entropy transfer did not affect the neutrinos because they were already completely decoupled, it is easy to calculate the change in the photon temperature before any $e^{ \pm}$annihilation and after the electron-positron pairs disappear by assuming entropy conservation of the electromagnetic plasma. Here, we quote the result between $T_{v}$ and $T_{v}$ [2]

$$
\frac{T_{\gamma}^{\text {affer }}}{T_{\gamma}^{\text {before }}}=\left(\frac{11}{4}\right)^{1 / 3} \simeq 1.40102=\frac{T_{\gamma}}{T_{v}}
$$

which is also the ratio between the temperatures of relic photons and neutrinos.

One can see in Figure 1 how in this epoch the photon temperature decreases with the expansion less than the inverse of the scale factor $a$. Instead the temperature of the decoupled neutrinos always falls as $1 / a$. Our interest is to study the electromagnetic process for the conversion $\gamma \rightarrow v+\bar{v} \Leftrightarrow e^{-}+e^{+}$at $T \geq 1 \mathrm{MeV}$. This paper is structured as follows. In Section 2 we start by reviewing the Dirac equation under chiral representation. In Section 3 we obtain the Dirac equation deduced from Maxwell's equations with $\boldsymbol{E} \| \boldsymbol{H}$ applied to neutrino at $T \geq 1 \mathrm{MeV}$ which can provide light neutrino masses under a chiral electromagnetic mechanism. Section 4 discusses the chiral electromagnetic neutrino.

\section{Chiral Dirac Neutrino Equation}

Plane-wave solutions of the Dirac equation are well known, and it would appear to be necessary to justify a reconsideration of this problem related with Dirac neutrino particles. The justification lies in the fact that the solutions found in journals and elsewhere do not lend themselves readily to a direct physical interpretation. In the usual treatment of the problem, four mutually orthogonal solutions are obtained for a given value of the momentum $p$, two of which correspond to positive energy and two to negative energy. However, the physical distinction between a pair of solutions belonging to the same energy does not appear until one considers the low energy approximation in which the small components of the state function are neglected and the two solutions reduce to eigenfunctions of $\sigma_{z}$ belonging to eigenvalues \pm 1 .

The original Dirac equation is

$$
\left(c \hat{\alpha} \cdot \hat{p}+m c^{2} \beta\right) \psi=i \hbar \frac{\partial \psi}{\partial t}
$$

where $\psi=\psi(\boldsymbol{r}, t)$ is a complex four-component field $\psi$ that Dirac thought of as the wave function for the electron, $\boldsymbol{r}$ and $t$ are the space and time coordinates, $m$ is the rest mass of the electron, $\hat{p}$ is the momentum operator, $c$ is the speed of light speed, and $\hbar$ is the reduced Planck constant Planck $(h / 2 \pi)$. Furthermore, $\hat{\alpha}$ is a vector operator whose components are $4 \times 4$ matrices: $\hat{\alpha}=\left(\alpha_{1}, \alpha_{2}, \alpha_{3}\right)$, and $\beta$ is another $4 \times 4$ matrix.

In the usual notation, the Dirac Hamiltonian for a free particle is [10]

$$
H=-c \hat{\alpha} \cdot \hat{p}-m c^{2} \beta .
$$

This Hamiltonian commutes with the momentum vector $\hat{p}$, and the usual procedure is to seek simultaneous eigenfunctions of $H$ and $p$. These eigenfunctions are, however, not uniquely determined, and for given eigenvalues of $H$ and $p$, there remains a twofold degeneracy. In order to resolve this degeneracy we seek a dynamical variable which commutes with both $H$ and $\hat{p}$. Such a variable is $\hat{\sigma} \cdot \hat{p}$, where $\hat{\sigma}$ is the matrix Pauli. It is obvious that this variable commutes with $\hat{p}$. To verify that it also commutes with $H$, we write $\hat{\alpha}=\rho_{1} \hat{\sigma}=\hat{\sigma} \rho_{1}$, and recalling that $\beta$ commutes with operator $\hat{\sigma}$, we have

$$
\left(c \rho_{1} \hat{\sigma} \cdot \hat{p}\right)(\hat{\sigma} \cdot \hat{p})-(\hat{\sigma} \cdot \hat{p})\left(c \rho_{1} \hat{\sigma} \cdot \hat{p}\right)=0,
$$

since $\rho_{1}$ commutes with $\hat{\sigma}$.

We now proceed to find simultaneous eigenfunctions of the commuting variables $H, p$ and $\hat{\sigma} \cdot \hat{p}$. We have, since the components of $p$ commute,

$$
(\hat{\sigma} \cdot \hat{p})^{2}=p^{2}
$$

where $p$ is the magnitude of the momentum vector. Thus for a simultaneous eigenstate of $\hat{p}$ and $\hat{\sigma} \cdot \hat{p}$, the value of $\hat{\sigma} \cdot \hat{p}$ will be $+p$ or $-p$, corresponding to states for which the spin is parallel or antiparallel, respectively, to the momentum vector.

A simultaneous eigenfunction of $H$ and $p$ will have the form of a plane wave [10]

$$
\psi_{j}(r, t)=\phi_{j} \exp [i(p \cdot r-W t) / \hbar], \quad j=1,2,3,4,
$$

where $\psi_{j}$ are the four components of the wave function and $\phi_{j}$ are four numbers to be determined. In the argument of the exponential function, $p$ represents the eigenvalues of the components of the momentum for this state and $W$ the corresponding eigenvalue of $H$. The possibility of positive mass and negative mass is formally consistent with the positive and negative solutions to the equations of the Special Theory of Relativity when combined with quantum mechanics [11]. Then $W$ can have either of the two values.

$$
W= \pm\left(m^{2} c^{4}+c^{2} p^{2}\right)^{\frac{1}{2}} .
$$

In Equation (5) $p$ is the relativistic momentum and $m$ is the invariant mass. There are two solutions to Equation (5); a positive solution and a negative solution. 
Dirac [11] described these as the "wanted" and "unwanted" solutions, respectively [12]. Pauli [13] considered that only the positive energy solutions had meaning. By contrast, here in connection with Dirac neutrino we interpret both the positive and negative energy solutions to be real solutions that represent substances with positive mass and negative mass, respectively.

Antimatter was first detected by observing the tracks left by positive electrons or positrons in cloud chambers caused by the movement of particles with an apparent charge-to-mass ratio $(\mathrm{e} / \mathrm{m})$ that was opposite in sign to that of electrons [14-16]. Related to Dirac neutrino, as we will show below, the anti Dirac neutrino could be described as a negative mass and uncharged particle. To do that, we now demand that $\psi_{j}$ be also an eigenfunction of $\hat{\sigma} \cdot \hat{p}$ belonging to one of the eigenvalues $p$, say, where $p \rightarrow \pm p$. Employing the usual matrix representation for $\hat{\sigma}$, we have

$$
\hat{\sigma} \cdot \hat{p}=\left(\begin{array}{cc}
p_{z} & p_{x}-i p_{y} \\
p_{x}+i p_{y} & -p_{z}
\end{array}\right) .
$$

In the above matrix, $p_{x}, p_{y}$ and $p_{z}$ are operators, but since this matrix is to operate on an eigenfunction of $p$, the operators can be replaced by their eigenvalues. We shall, without risk of confusion, use the same symbols for the eigenvalues as for the corresponding operators.

The eigenvalue equation

$$
\hat{\sigma} \cdot \hat{p} \psi=p \psi,
$$

yields the following four equations:

$$
\begin{aligned}
& p_{z} \phi_{1}+\left(p_{x}-i p_{y}\right) \phi_{2}=p \phi_{1} \\
& \left(p_{x}+i p_{y}\right) \phi_{1}-p_{z} \phi_{2}=p \phi_{2} \\
& p_{z} \phi_{3}+\left(p_{x}-i p_{y}\right) \phi_{4}=p \phi_{3} \\
& \left(p_{x}+i p_{y}\right) \phi_{3}-p_{z} \phi_{4}=p \phi_{4}
\end{aligned}
$$

These equations are satisfied by

$$
\begin{array}{ll}
\phi_{1}=p_{x}-i p_{y}, & \phi_{2}=p-p_{z}, \\
\phi_{3}=\chi\left(p_{x}-i p_{y}\right), & \phi_{4}=\chi\left(p-p_{z}\right),
\end{array}
$$

where $\chi$ is a number still to be determined (The consistency of the equations is readily verified, recalling that $p \rightarrow \pm p$ ).

We now make use of the requirement that $\psi$ be an eigenfunction of $H$ belonging to the eigenvalue $W$.

Then

$$
(W-H) \psi=0
$$

that is,

$$
\left(W+c \rho_{1} \hat{\sigma} \cdot \hat{p}+m c^{2} \beta\right) \psi=0 .
$$

In terms of chiral matrices $\hat{\alpha}-\beta$, in Equation (11) we replace $\hat{\sigma} \cdot \hat{p}$ by its eigenvalue to obtain

$$
\left(W+c p \rho_{1}+m c^{2} \beta\right) \psi=0 .
$$

On expansion, we obtain

$$
\begin{aligned}
& 0=(W+c p) \phi_{1}+m c^{2} \phi_{2} \\
& 0=(W-c p) \phi_{2}+m c^{2} \phi_{1} \\
& 0=(W+c p) \phi_{3}+m c^{2} c \pi \phi_{4} \\
& 0=(W-c p) \phi_{4}+m c^{2} \phi_{3}
\end{aligned}
$$

This system of equations is the chiral Dirac equation

$$
i \gamma_{c h}^{\mu} \partial_{\mu} \psi-(m c / \hbar) \psi=0
$$

The condition $E= \pm W$ ensures the consistency of these equations. Comparing Equation (13) with Equation (9), we find

$$
\chi=-\frac{W+c p}{m c^{2}}=-\frac{m c^{2}}{W-c p} .
$$

We can now write the components of $\psi$ as follows:

$$
\psi_{j}=\phi_{j} \exp [i(p \cdot r-W t) / h],
$$

where $\phi_{j}$ is given by

$$
\begin{aligned}
& \phi_{1}=p_{x}-i p_{y}, \\
& \phi_{2}=p-p_{z}, \\
& \phi_{3}=-(W-c p)\left(p_{x}-i p_{y}\right) / m c^{2}, \\
& \phi_{4}=-(W-c p)\left(p-p_{z}\right) / m c^{2} .
\end{aligned}
$$

Since $W$ can be given either of the two values $\pm W$ and $p$, the two values $\pm p$, we have found for given $p$ four linearly independent plane wave solutions. It is easily verified that they are mutually orthogonal.

The physical interpretation of the solutions is now clear. Each solution represents a homogeneous beam of Dirac neutrino particles of definite momentum $p$, of definite energy, either $\pm W$, and with the spin polarized either parallel or antiparallel to the direction of propagation. This condition can be obtained if we consider the Maxwell's equations under a chiral approach [17-19]. Solutions $\phi_{j}$ of Equations (9) and (16) are still undetermined because we need to link them with chiral Maxwell equations. In the next section, solutions $\phi_{j}$ will be related with the chiral electrodynamics when $\boldsymbol{E} \| \boldsymbol{H}$.

\section{Dirac Neutrino Equation Deduced from Maxwell's Equations with $E \| H$}

Here we show that it is possible to transform the Maxwell equation from six real components to four complex components and in this form to obtain two spinor equations in the Weyl or chiral representation. First, we will 
consider the Maxwell equations for a sourceless anisotropic chiral homogeneous medium. Following [20,21], with the time variation as: $\delta_{t}=\partial_{t}\left(1+T_{c} \nabla \times\right)$ and $T_{c}$ is as a chiral parameter, the Maxwell's equations without charges are expressed as:

$$
\begin{gathered}
\operatorname{rot} \boldsymbol{H}=\frac{1}{c} \partial_{t}\left(1+T_{c} \nabla \times\right) \boldsymbol{E}, \\
\operatorname{div} \boldsymbol{E}=0 \\
\operatorname{rot} \boldsymbol{E}=-\frac{1}{c} \partial_{t}\left(1+T_{c} \nabla \times\right) \boldsymbol{H}, \\
\operatorname{div} \boldsymbol{H}=0
\end{gathered}
$$

Here, $\operatorname{rot}=\nabla \times, \omega$ is the frequency and $c$ is the light velocity. Application of rot to (17) and (19) allows us to write the wave equation for $\mathbb{F}=\boldsymbol{E}$, or $\boldsymbol{H}$.

$$
\begin{aligned}
& \operatorname{rot}(\operatorname{rot} \mathbb{F})+\frac{1}{c^{2}} \partial_{t}^{2} \mathbb{F}+\frac{2 T_{c}}{c^{2}} \partial_{t}^{2} \operatorname{rot} \mathbb{F} \\
& +\frac{T_{c}^{2}}{c^{2}} \partial_{t}^{2} \operatorname{rot}(\operatorname{rot} \mathbb{F})=0 .
\end{aligned}
$$

This chiral wave equation represents an equation of fourth order. Here we consider the condition

$$
\left(1+\frac{T_{c}^{2}}{c^{2}} \frac{\partial^{2}}{\partial t^{2}}\right)=0
$$

where Equation (21) is transformed from a fourth order equation to a first order one.

$$
\mathbb{F}+2 T_{c}(\operatorname{rot} \mathbb{F})=0
$$

and the electric field is parallel to the magnetic field $\boldsymbol{E}=i \boldsymbol{H}$. The linear Beltrami Equation (23), will be used to construct the Dirac neutrino equation in chiral or Weyl representation.

In [14], it was shown that the ordinary Dirac equation is equivalent to the chiral quaternionic equation

$$
D_{\boldsymbol{\alpha}_{c}} \psi=\left(D+\boldsymbol{\alpha}_{c}\right) \psi=0
$$

with $\boldsymbol{\alpha}_{c}:=\left(i \omega c^{-1} \sigma_{1}-m_{D} c \hbar^{-1} \sigma_{3}\right)$ and the Maxwell Equations (17)-(22) are equivalent to the chiral Equation (23) when the electric field $\boldsymbol{E}$ is parallel to the magnetic field $\boldsymbol{H}(\boldsymbol{E}=i \boldsymbol{H})$. Multiplying Equation (23) by $i \hat{\sigma}$ we have

$$
\begin{gathered}
i \hat{\sigma} \cdot \mathbb{F}+2 T_{c}(i \hat{\sigma} \cdot \operatorname{rot} \mathbb{F})=0 \\
\text { that is, with } \nabla \cdot \mathbb{F}=0 \\
i \hat{\sigma} \cdot \mathbb{F}+2 T_{c} \hat{\sigma} \cdot \nabla(\hat{\sigma} \cdot \mathbb{F})=0 .
\end{gathered}
$$

From this equation we can separate

$$
\begin{aligned}
& i \hat{\sigma} \cdot \boldsymbol{E}+2 T_{c} \hat{\sigma} \cdot \nabla(\hat{\sigma} \cdot \boldsymbol{E})=0, T_{c}>0 \\
& i \hat{\sigma} \cdot \boldsymbol{H}+2 T_{c} \hat{\sigma} \cdot \nabla(\hat{\sigma} \cdot \boldsymbol{H})=0, T_{c}<0
\end{aligned}
$$

$$
\text { Let } \phi_{R}=(\hat{\sigma} \cdot i E) \mathrm{I}, \phi_{L}=(\hat{\sigma} \cdot i H) \mathrm{I}, \quad \mathrm{I}=\left(\begin{array}{ll}
1 & 0
\end{array}\right)^{\mathrm{T}},
$$

and making $\partial_{t}=i \omega, \omega T_{c} / c= \pm 1$ and $T_{c}= \pm \hbar / 2 m c$ we obtain

$$
\begin{gathered}
i \hbar\left(c^{-1} \partial_{0}+\hat{\sigma} \cdot \nabla\right) \phi_{R}=m c \phi_{L} \\
i \hbar\left(c^{-1} \partial_{0}-\hat{\sigma} \cdot \nabla\right) \phi_{L}=m c \phi_{R} .
\end{gathered}
$$

Equations (24) and (25) are equivalent to the quarternionic chiral Dirac equation obtained in reference [14], here $m=m_{D}$ is the Dirac neutrino mass. Solutions of (24) and (25) may be called the Beltrami fields (see, e.g., [21]). R and L, subscripts are associated with circularly polarized photons (right or left-handed spirals or chiral photons), that is photons that have closed trajectories to form standing waves that generate mass. The Dirac neutrino equation in chiral or Weyl representation is [15],

$$
i \gamma_{c h}^{\mu} \partial_{\mu} \psi-(m c / \hbar) \psi=0
$$

In the chiral Dirac neutrino field $\psi=\left(\phi_{R}, \phi_{L}\right)^{\mathrm{T}}, \phi_{R}$ and $\phi_{L}$ are two different types of 2-component spinor, they respectively correspond to the nonequivalent representations $(1 / 2,0)$ and $(0,1 / 2)$ of the Lorentz group, and the chiral Dirac neutrino equation presents a relation between the two spinors. If we consider parity, then it is no longer sufficient to consider the 2-component spinors $\phi_{R}$ and $\phi_{L}$ separately, but the 4-component spinor $\psi=\left(\phi_{R}, \phi_{L}\right)^{\mathrm{T}}$. This 4-component spinor is an irreducible representation of the Lorentz group extended by parity (i.e., the $(1 / 2,0) \oplus(0,1 / 2)$ representation).

Now we are in conditions of compare Equation (13') with Equation (26) and show that the dirac neutrino particle with mass generated by the $\boldsymbol{E} \| \boldsymbol{H}$ configuration.

$$
\psi=\left(\begin{array}{c}
E_{x}-E_{y} \\
|\boldsymbol{E}|-E_{z} \\
-\frac{|\boldsymbol{E}| c}{W-m c^{2}}\left(E_{x}-E_{y}\right) \\
-\frac{|\boldsymbol{E}| c}{W-m c^{2}}\left(|E|-E_{z}\right)
\end{array}\right)
$$
where the electric field is given by $|\boldsymbol{E}|=\frac{W m c}{2 \pi e \hbar}$ and
$W=\hbar \omega$.

As $T_{c}$ is positive or negative we can generalize the product $p T_{c}$ as $p T_{c}= \pm \hbar / 2$. For a massive Dirac neutrino, the electric and magnetic fields are $90^{\circ}$ out of phase, the energy density is constant and proportional to

$$
4 \pi G\left(\boldsymbol{E}^{2}+\eta^{2} \boldsymbol{H}^{2}\right) \sim 4 \pi k^{2} F_{0}^{2} .
$$

We find in SI units, that $4 \pi k^{2} F_{0}^{2}=\left(1.3 \times 10^{-9} E E_{c}^{-1} m^{-1}\right)^{2}$. Here $E_{c}$ is the critical field for neutrino-antineutrino pair equivalent to the electron-positron pair production given by 


$$
E_{c}=m_{e}^{2} c^{3} e^{-1} \hbar^{-1} \sim 1.3 \times 10^{18} \mathrm{Vm}^{-1} .
$$

That is equivalent to $1.3 \times 10^{12} \mathrm{G}$. Observations indicate that neutron stars have magnetic fields higher than $10^{12} \mathrm{G}$ so neutrino-antineutrino pair is generated. In this form we have a close connection between the Dirac neutrino equation and the Maxwell's equation, with a direct and complete physical interpretation in the $\boldsymbol{E} \| \boldsymbol{H}$ configuration. Here we are obtained a clear connection between the Planck constant $\hbar$, the quiral factor $T_{c}$, and the electromagnetic mass which can be positive or negative according to $T_{c}$.

It is well known that matter and antimatter respond to electromagnetic radiation in opposite ways. For example, if an electron (Dirac neutrino) moves one way in an electromagnetic field, a positron (Dirac anti neutrino) will move in the opposite way. We apply our theory of positive and negative mass to matter and antimatter and suggest that it is productive to consider matter as having a positive mass (+chirality) and antimatter as having a negative mass (-chirality). The equations presented here, which treat matter as having a positive mass and antimatter as having a negative mass, can account for the experimental observations of matter and antimatter in chiral electromagnetic fields. Our treatment allows the symmetry between matter and antimatter to be treated in a more causal manner.

Taking into consideration the possibility of negative mass, we can describe the observed behavior of antimatter in response to electromagnetic fields by assuming that the positron (anti Dirac neutrino) has a negative mass. Given that particles and antiparticles respond oppositely to an input of various forms of electromagnetic energy, we considered the possibility that the mass can be considered in a causal symmetry. Taking into account the concept of relative time, particularly the relativity of the direction of time to describe antimatter, we now suggest a more causal symmetry between matter and antimatter. That is, instead of using CPT symmetry [22], where matter is antisymmetrical with antimatter in terms of its charge $(C)$, parity $(P)$ and direction in time $(T)$, we suggest that matter is antisymmetrical with antimatter in terms of its mass, parity and time.

$$
M_{\text {part }} P T=-M_{\text {conjantipart }} P T \text {. }
$$

So the combined MPT operations leaves invariant the Dirac Equation (1)

$$
\begin{aligned}
& M_{\text {particle }} P T: \\
& \left(c \hat{\alpha} \cdot \hat{p}+m c^{2} \beta\right) \psi=i \hbar \frac{\partial \psi}{\partial t} \\
& \rightarrow\left(c \hat{\alpha} \cdot(-\hat{p})+(-m) c^{2} \beta\right) \psi=i \hbar \frac{\partial \psi}{\partial(-t)}
\end{aligned}
$$

In the chiral representation also can be show that the Dirac neutrino equation is invariant.

\section{Chiral Electromagnetic Neutrino}

Dirac introduced the concept of antiparticles, [23]. Now we know that for every particle there is an antiparticle.

However some particles could be self-conjugate, in the sense that particle and antiparticle could be the same. Of course such particles have to be electrically neutral.

The possibility of a self-conjugate fermion was first pointed out by Majorana [24], and hence they are called Majorana fermions while the other fermions (with distinct particles and antiparticles) are called Dirac fermions, Equations (24) and (25) [25]. Among the fermions of the Standard Model, only neutrinos are electrically neutral and hence qualify to be Majorana particles. But it is still an open question whether neutrinos are Majorana particles or Dirac particles. In our theory we can discriminate this situation if $T_{c}=\hbar / 2 m c \sigma_{1}$ with $m=m_{M R, L}$ or $T_{c}=\hbar / 2 m c \sigma_{3}$ with $m=m_{D}$ respectively.

If neutrinos are Majorana particles, lepton number $L$ is not conserved and this opens the door to generate an excess of leptons over antileptons in the early universe which can subsequently generate an excess of baryons over antibaryons, thus explaining how after annihilation of most of the particles with antiparticles, a finite but small residue of particles was left, to make up the present Universe.

Equations (24) and (25) support the see-saw mechanism to explain why the neutrino masses, although not zero, are so tiny.The seesaw mechanism is a generic model used to understand the relative sizes of observed neutrino masses, of the order of eV. See-saw mechanism maybe the reason for the smallness of the masses.

*Provides an explanation for the smallness of the neutrino masses;

$* m_{v L}=0$ is natural, since a $v_{L}$ mass term is forbidden by the SM symmetries;

* $m_{D}$, generated by the Higgs mechanism can be taken the order of the charged lepton masses;

* $m_{R}$ is not "protected" by the SM symmetries, and can be taken large.

With our theory, using Equations (24) and (25) it is possible to obtain a matrix mass

$$
\left(\begin{array}{ll}
m_{v L} & m_{D} \\
m_{D} & m_{v R}
\end{array}\right) .
$$

If $m_{v} \sim 1 \mathrm{eV}$ with $m_{R} \gg m_{D}$ we obtain two Majorana particles, an electron neutrino light $(\sim 1 \mathrm{eV})$ and a sterile heavy one $\left(m_{R} \sim 1 \mathrm{TeV}\right)$. In addition to the nonzero neutrino masses and mixing angles, the nonzero magnetic moment $\mu_{v}$ is another property of neutrinos beyond the standard model of particle physics. The im- 
portance of $\mu_{v}$ was first mentioned by Pauli [18]. Systematic theoretical studies of neutrino electromagnetic properties started after it was shown that in the extended Standard Model with right-handed neutrinos the magnetic moment of a massive neutrino is, in general, nonvanishing and that its value is determined by the neutrino mass [26].

If neutrinos have a nonzero magnetic moment, it leads to precession between left and right-handed neutrinos in sufficiently strong magnetic fields [27]. In general, nondiagonal elements of the magnetic moment matrix are possible and neutrinos can be changed into different flavours and chiralities, see Equations (24) and (25). Furthermore, with the additional effect of coherent forward scattering by matter, neutrinos can be resonantly converted into those with different chiralities.

For a neutrino mass smaller than $1 \mathrm{eV}$, a Dirac neutrino has a non-zero magnetic moment proportional to the neutrino mass, that yields a very small value for the magnetic moment, $\mu_{v} \sim 3 \times 10^{-19} \mu_{\beta}$. As $\mu_{\beta}=e \hbar / 2 m$. From our theory, the Bohr magneton is proportional to $T_{c}$ so in this case we have $T_{c}=\mu_{\beta} / e c \sim 10^{-8} \mathrm{~m}$ ). From data obtained of astrophysics (considering the cooling of red giant stars), we obtain

$$
T_{c} \leq 3 \times 10^{-12} \mu_{\beta} / 3 \times 10^{-11} \sim 0.1 \mathrm{~m}
$$

corresponding to neutrino $\mu_{v}$. There is a gap of some orders of magnitude between the present experimental limits $\sim 10^{-11} \mu_{\beta}-10^{-12} \mu_{\beta}$ [28], on neutrino magnetic moments and the predictions of different extensions of the Standard Model which hint at a range $\sim 10^{-14} \mu_{\beta}-10^{-15} \mu_{\beta} \quad$ [29]. The main problem in distinguishing Dirac from Majorana neutrino is the lack of neutrino with positive helicity. One way is to reverse the spin of the neutrino in an external magnetic field. The problem is that we need large neutrino magnetic moments and large magnetic field to obtain visible effects. However with our theory it is possible to reverse the spin using laser technology to have sufficient energy density to generate Dirac neutrino [30].

$$
4 \pi G\left(E^{2}+\eta^{2} H^{2}\right) \sim\left(1.3 \times 10^{-9} E E_{e-i} m^{-1}\right)^{2} .
$$

Here $E_{e-i}$ is the critical field for electron positron production $E_{e-i} \sim 1.3 \times 10^{18} \mathrm{Vm}^{-1}$. We think that by measuring the chirality $T_{c}$ of the processes, we may have an improvement in the study of electromagnetic properties of neutrinos.

An example is the modeling of neutrinos propagation during core-collapse supernovae where very strong magnetic fields are believed to exist and in which the influence of neutrino electromagnetic properties has not yet been taken into account. Equation (24) is well suited to study the electromagnetic properties of neutrinos and
Majorana particles such as charge conjugation and time reversal, allowing for an experimental study of our Equations (24)-(26).

\section{Conclusions}

The two-component Dirac neutrino equation, coming in two related forms (24) and (25) that are connected by a spin flip, represents the simplest possible covariant relativistic wave equation for a massive fermion. These two forms represent the two irreducible representations of the Lorentz group in terms of Pauli spinors and matrices. Also the Dirac neutrino equation can directly be derived by linearization of Equation (8), a procedure which leads to (24) and (25) without explicit recourse to the Dirac theory. An important characteristic of neutrino electromagnetic properties is that they are different from Dirac and Majorana neutrinos. In particular, Majorana neutrinos cannot have diagonal magnetic or electric moments. Thus, studies of neutrino electromagnetic interactions from Equations (24) and (25) can be used as a procedure to distinguish whether a neutrino is a Dirac or Majorana particle.

Moreover, CP invariance in the lepton sector puts additional constraints on the neutrino form factors and can be tested with experimental probes of neutrino electromagnetic interactions.

\section{REFERENCES}

[1] G. Drexlin, et al., "Current Direct Neutrino Mass Experiments," Advances in High Energy Physics Vol. 2013, 2013, Article ID: 293986. doi:10.1155/2013/293986

[2] J. Lesgourgues and S. Pastor, "Massive Neutrinos and Cosmology," Physics Reports, Vol. 429, No. 6, 2006, pp. 307-379. doi:10.1016/j.physrep.2006.04.001

[3] A. Cho, "The Sterile Neutrino: Fertile Concept or Dead End?" Science, Vol. 334, No. 6054, 2011, pp. 304-306. doi:10.1126/science.334.6054.304

[4] D. V. Forero, M. Tortola and J. W. F. Valle, "Global Status of Neutrino Oscillation Parameters after Neutrino2012," Physical Review D, Vol. 86, No. 7, 2012, Article ID: 073012.. doi:10.1103/PhysRevD.86.073012

[5] G. L. Fogli, E. Lisi, A. Marrone, D. Montanino, A. Palazzo and A. M. Rotunno, "Global Analysis of Neutrino Masses, Mixings, and Phases: Entering the Era of Leptonic CP Violation Searches," Physical Review D, Vol. 86, No. 1, 2012, Article ID: 013012.

[6] M. C. Gonzalez-Garcia and M. Maltoni, "Phenomenology with Massive Neutrinos," Physics Reports, Vol. 460, No. 1-3, 2008, pp. 1-129. doi:10.1016/j.physrep.2007.12.004

[7] A. G. Cohen, S. L. Glashow and Z. Ligeti, "Disentangling Neutrino Oscillations," Physics Letters B, Vol. 678, No. 2, 2009, pp. 191-196. doi:10.1016/i.physletb.2009.06.020

[8] Z. Maki, M. Nakagawa and S. Sakata, "Remarks on the Unified Model of Elementary Particles," Progress of 
Theoretical Physics, Vol. 28, No. 5, 1962, pp. 870-880. doi:10.1143/PTP.28.870

[9] J. W. F. Valle, "Neutrino Physics Overview," Journal of Physics: Conference Series, Vol. 53, No. 1, 2006, p. 473. doi:10.1088/1742-6596/53/1/031

[10] S. M. Neamtan, "On the Physical Interpretation of Solutions of the Dirac Equation for a Free Particle," American Journal of Physics, Vol. 20, No. 7, 1952, pp. 450-451. doi:10.1119/1.1933275

[11] P. A. M. Dirac, "A Theory of Electrons and Protons," Proceedings of the Royal Society A, Vol. 126, No. 801, 1930, pp. 360-365. doi:10.1098/rspa.1930.0013

[12] P. A. M. Dirac, "Theory of Electrons and Positrons," Nobel Lecture, December 12, 1933.

[13] W. Pauli, "The Connection between Spin and Statistics," Physical Review, Vol. 58, No. 8, 1940, pp. 716-730. doi:10.1103/PhysRev.58.716

[14] C. D. Anderson, "The Apparent Existence of Easily Deflectable Positives," Science, Vol. 76, No. 1967, 1932, pp. 238-239. doi:10.1126/science.76.1967.238

[15] C. D. Anderson, "The Positive Electron," Physical Review, Vol. 43, 1933, pp. 491-494. doi:10.1103/PhysRev.43.491

[16] C. D. Anderson, "The Discovery of Antimatter," World Scientific, Singapore, 1999.

[17] C. Chu and T. Ohkawa, "Transverse Electromagnetic Waves with E B" Physical Review Letters, Vol. 48, 1982, pp. 837-838. doi:10.1103/PhysRevLett.48.837

[18] H. Zaghloul, H. A. Buckmaster and K. Volk, "Comment on "Invariants of the Electromagnetic Field and Electromagnetic Waves,," American Journal of Physics, Vol. 56, No. 3, 1988, p. 274. doi:10.1119/1.15664

[19] H. Torres-Silva, "Chiral Transverse Electromagnetic Standing Waves with E II H in the Dirac Equation and the Spectra of the Hydrogen Atom," In: A. Akdagli, Ed., Behavior of Electromagnetic Waves in Different Media and Structures, Book Intech, 2011, pp. 301-324.
[20] H. Torres-Silva and D. Torres, "Chiral Current in a Graphene Battery," Journal of Electromagnetic Analysis and Applications, Vol. 4, No. 10, 2012, pp 426-431. doi:10.4236/jemaa.2012.410059

[21] H. Torres-Silva, "Chiral Dirac Equation Derived from Quaternionic Maxwell's Systems," Journal of Electromagnetic Analysis and Applications, Vol. 5, No. 3, 2013. doi:10.4236/jemaa.2013.53017

[22] C. Itzykson and J.-B. Zuber, "Quantum Field Theory," McGraw-Hill, New York, 1980.

[23] P. M. A. Dirac, "The Quantum Theory of the Elec tron," Proceedings of the Royal Society of London. Series A, Containing Papers of a Mathematical and Physical Character, Vol. 117, No. 778, 1928, pp. 610-617.

[24] E. Majorana, “Teoria Simmetrica Dell' Elettrone E Del Positrone," Il Nuovo Cimento, Vol. 14, No. 4, 1937, pp. 171-184. doi:10.1007/BF02961314

[25] W. Pauli, "Zur Quantenmechanik des Magnetischen Elektrons," Zeitschrift für Physik A Hadrons and Nuclei, Vol. 43, No. 9-10, 1927, pp. 601-623. doi:10.1007/BF01397326

[26] C. Giunti and C. W. Kim, "Fundamentals of Neutrino Physics and Astrophysics," Oxford University Press, Oxford, 2007.

[27] S. Bilenky, "Introduction to the Physics of Massive and mixed Neutrinos," Lecture Notes in Physics, Vol. 817, 2010. doi:10.1007/978-3-642-14043-3

[28] N. F. Bell, et al., "How Magnetic Is the Dirac Neutrino?" Physical Review Letters, Vol. 95, No. 15, 2005, Article ID: 151802. doi:10.1103/PhysRevLett.95.151802

[29] A. Studenikin, "Neutrino Magnetic Moment," Nuclear Physics B, Vol. 188, 2009, pp. 220-222. doi:10.1016/j.nuclphysbps.2009.02.053

[30] E. Fermi and C. N. Yang, "Are Mesons Elementary Particles?” Physical Review, Vol. 76, No. 12, 1949, pp. 1739 1743. doi:10.1103/PhysRev.76.1739 